\title{
Identification of Peptide Antagonists to Thioredoxin Glutathione Reductase of Schistosoma japonicum
}

\author{
Li-Jun Song $\mathbb{D},{ }^{1,2}$ Jia-Huang Li $\mathbb{D},{ }^{1,3}$ Xu-Ren Yin $\mathbb{D},{ }^{2}$ Wei Zhang $\mathbb{D}^{2},{ }^{2}$ Yi Jin $\mathbb{D},{ }^{2}$ Hong Gao $\mathbb{D},{ }^{4}$ \\ Jie Wang $\mathbb{D}^{2},{ }^{2}$ Chuan-Xin Yu $\mathbb{D},^{2}$ and Zi-Chun Hua $\mathbb{D}^{1,3,5}$ \\ ${ }^{1}$ School of Life Sciences and the State Key Laboratory of Pharmaceutical Biotechnology, Nanjing University, Nanjing, \\ Jiangsu Province 210023, China \\ ${ }^{2}$ Key Laboratory of National Health and Family Planning Commission on Parasitic Disease Control and Prevention, Jiangsu Provincial \\ Key Laboratory on Parasite and Vector Control Technology, Jiangsu Institute of Parasitic Diseases, Wuxi, Jiangsu Province 214064, \\ China \\ ${ }^{3}$ Changzhou High-Tech Research Institute of Nanjing University and Jiangsu Target Pharma Laboratories Inc., Changzhou, \\ Jiangsu Province 213164, China \\ ${ }^{4}$ Department of Pathology, Nanjing Drum Tower Hospital, The Affiliated Hospital of Nanjing University Medical School, Nanjing, \\ Jiangsu Province 210008, China \\ ${ }^{5}$ Shenzhen Research Institute of Nanjing University, Shenzhen, Guangdong Province 518057, China
}

Correspondence should be addressed to Chuan-Xin Yu; chxnyu@163.com and Zi-Chun Hua; huazc@nju.edu.cn

Received 16 January 2018; Revised 23 March 2018; Accepted 3 April 2018; Published 5 June 2018

Academic Editor: Rita Casadio

Copyright (C) 2018 Li-Jun Song et al. This is an open access article distributed under the Creative Commons Attribution License, which permits unrestricted use, distribution, and reproduction in any medium, provided the original work is properly cited.

\begin{abstract}
Schistosomiasis is one of the world's major public health problems. Praziquantel is currently the only effective drug against schistosomiasis. As resistance of praziquantel has emerged in some endemic areas, development of new antischistosomal agents should be a high priority. In this study, a phage display peptide library was used for screening for peptide antagonists of thioredoxin glutathione reductase of Schistosoma japonicum (SjTGR), which has been identified as an alternative drug target. Three rounds of panning produced four different fusion phages. ELISA proved that all four phages could bind to SjTGR. One peptide, JIPDys1 (aa, WPHNWWPHFKVK), reduced enzyme activity of SjTGR by more than $50 \% .2 \mu \mathrm{M}$ of the synthesized peptide of JIPDysl inhibited the activity of TrxR, GR, and Grx of SjTGR by $32.5 \%, 100 \%$, and 100\%, respectively. The $\mathrm{IC}_{50}$ values of the synthetic peptide JIPDys1 for $\operatorname{TrxR}$, GR, and Grx were $3.67 \mu \mathrm{M}, 0.11 \mu \mathrm{M}$, and $0.97 \mu \mathrm{M}$, respectively. Based on computer simulation, it appeared that JIPDys1 binds to the substrate binding sites of glutathione reductase (GR) and glutaredoxin (Grx). Our data show that the peptide, JIPDys1 (aa, WPHNWWPHFKVK), is a promising candidate to develop novel drugs against S. japonicum which acts by binding with SjTGR and reduces enzyme activity of SjTGR.
\end{abstract}

\section{Introduction}

Schistosomiasis, a serious disease caused by intravascular trematodes of the genus Schistosoma, is one of the world's major public health problems [1-4]. Adult parasites reside in the mesenteric veins of their human hosts, surviving on the host's red blood cells, which results in anemia, diarrhea, splenomegaly, liver fibrosis, and other symptoms [5]. The drug of choice, praziquantel (PZQ) [6, 7], is administered globally to 100 million people each year; but sensitivity problems are emerging. Decreased sensitivity of Schistosoma mansoni and Schistosoma haematobium to praziquantel has been reported in some endemic areas [8-14]. Artemisinin, which was developed as antimalaria drug, seems to be active against Schistosoma [15]. However, juvenile worms are more sensitive to the drug than adult worms [15]. As there is currently no other effective treatment against schistosomiasis, development of new antischistosomal agents to curb the emergence of drug-resistance should be a high priority.

Organisms are continuously attacked by reactive oxygen species (ROS). There are two major systems existing to detoxify ROS in eukaryotes, the thioredoxin (Trx) system, 
and the glutathione (GSH) system, depending on the Trx reductase (TrxR) and GSH reductase (GR), respectively [16]. However, it has been reported that thioredoxin glutathione reductase (TGR) in plathelminthes such as S. mansoni [1720], S. japonicum [21], larval Taenia crassiceps (cysticerci) [22], Echinococcus granulosus [23], and Fasciola hepatica [24] has replaced the separate enzymes TrxR and GR and plays a critical role in thiol-disulfide redox homeostasis. Our initial work using recombinant S. japonicum TGR [21] suggested that TGR was essential for $S$. japonicum survival and suited as potential target for development of novel drugs against $S$. japonicum.

Techniques using library screening have improved other methods $[19,25]$ to identify components as potential drugs against diseases. These new methods have hugely increased speed and throughput to identify interesting lead candidates. In the present study, one of such methods, phage display peptide library screening, was used to identify peptide antagonists to SjTGR. Computer simulation and molecular docking were used to analyze interacting sites of the phage display peptide with SjTGR. The results show that a novel peptide inhibited SjTGR activity likely by preventing the binding of substrates to SjTGR. Our findings lay the foundation for the further development of peptide drugs against the activity of SjTGR.

\section{Materials and Methods}

2.1. Phage Display Peptide Library Screening with Recombinant SjTGR. The purchased Ph.D-12 phage display peptide library kit (New England Biolabs, Massachusetts, USA) contained $1 \times 10^{13} \mathrm{pfu} / \mathrm{ml}$ with a complexity of $1.0 \times 10^{12}$ transformants and used filamentous coliphage M13 for peptide expression. Phage was propagated in Escherichia coli strain ER2738 provided with the kit. 3,3,5,5'-Tetramethylbenzidine (TMB) substrate solution was purchased from Neobioscience Technology Company Limited (Beijing, China) and horseradish peroxidase (HRP) conjugated anti-M13 monoclonal antibody from GE Healthcare Life Sciences (Piscataway, NJ, USA).

Recombinant SjTGR protein expression and purification was described previously [21]. $1.5 \mathrm{ml}$ of a $100 \mu \mathrm{g} / \mathrm{ml}$ solution of SjTGR in $0.1 \mathrm{M} \mathrm{NaHCO} 3(\mathrm{pH} 8.6$ ) was added per well to a 12-well plate (Corning Corporation, USA) and incubated at $4^{\circ} \mathrm{C}$ overnight. Following six washes with Tris-HCL buffered saline (50 mM Tris, $150 \mathrm{mM} \mathrm{NaCl}, \mathrm{pH} 7.5$ ) containing $0.1 \%$ Tween-20 (TBST), the well was blocked with $400 \mu \mathrm{l} 5 \mathrm{mg} / \mathrm{ml}$ Bovine Serum Albumin (BSA) in Tris-HCL buffered saline (TBS) and incubated for 1 hour (h) at $4^{\circ} \mathrm{C}$. Then, $2 \times 10^{11}$ $\mathrm{pfu}$ of the phages was added for $1 \mathrm{~h}$ at room temperature (rt) with shaking. After six washes with TBST, the bound phages were eluted with $0.5 \mathrm{mM}$ NADPH and $1 \mu \mathrm{l}$ of phage solution diluted with LB medium was used to determine the phage titer. The remaining eluate was amplified by infecting $20 \mathrm{ml}$ of a 1/100 dilution of an overnight culture of E. coli ER2738, as recommended in the instruction manual. The amplified phages were used for the next round of panning, which was repeated twice. The same number of phage particles $\left(2 \times 10^{11}\right.$ pfu) was used in each round. The concentration of Tween-20 for washing was $0.1 \%$ for the first panning and $0.5 \%$ for the second and third panning.

2.2. DNA Sequencing of the Selected Phages Binding with SjTGR. Single phage plaques derived from the third round of panning were amplified and genomic DNA was extracted following the manual. The nucleotide sequences of the inserted peptides (Sangon Corporation, Shanghai, China) were obtained using -96 gIII sequencing primer, $5^{\prime}$ CCTCATAGTTAGCGTAACG- $3^{\prime}$, and -28 gIII sequencing primer, $\quad 5^{\prime}$-GTATGGGATTTTGCTAAACAAC- $3^{\prime}$. The amino acid (aa) sequence was deduced from the nucleotide sequence and compared with DNAman software (Version $6.0)$.

2.3. Phage Binding to Recombinant SjTGR by ELISA. Phage clones were amplified according to the manual. A 96-well plate was coated with $100 \mu \mathrm{l}$ of SjTGR solution $(10 \mu \mathrm{g} / \mathrm{ml}, 0.1$ $\mathrm{M} \mathrm{NaHCO} 3, \mathrm{pH}$ 8.6) overnight at $4^{\circ} \mathrm{C}$. After blocking with PBS containing 5\% milk powder at $\mathrm{rt}$ for $1 \mathrm{~h}, 100 \mu \mathrm{l}$ of phage solution with titers of $1 \times 10^{12}, 2.5 \times 10^{11}, 6 \times 10^{10}, 1.5 \times 10^{10}$, and $3.75 \times 10^{9} \mathrm{pfu} / \mathrm{ml}$ was used per well, respectively. The phage library was set as the negative phage control. Following an incubation at $37^{\circ} \mathrm{C}$ for $2 \mathrm{~h}$ and six washes with TBST (0.5\% Tween-20), $200 \mu \mathrm{l}$ of diluted HRP-conjugated antiM13 monoclonal antibody solution was added for $1 \mathrm{~h}$ at $\mathrm{rt}$. Following six washes with TBST, $100 \mu \mathrm{l}$ of substrate TMB solution was added for 10 minutes at rt. $50 \mu \mathrm{l}$ of $2 \mathrm{M}$ sulfuric acid solution $\left(\mathrm{H}_{2} \mathrm{SO}_{4}\right)$ was added to terminate the reaction and absorbance at $450_{\mathrm{nm}}$ was measured. OD $450_{\mathrm{nm}}$ values 2.1 times above the negative control value were considered positive.

2.4. Activity of the Isolated Phages and Synthetic Peptides. To assess the inhibitory action of phages, the activities of TrxR, GR, and glutaredoxin (Grx) of SjTGR were assayed as described in [21]. $10 \mu \mathrm{l}\left(10^{14} \mathrm{pfu} / \mathrm{ml}\right)$ of phage solution was added to the reaction system, phage library was used as negative phage control, and the effect of phages on the activity of SjTGR was expressed as percentage inhibition: Inhibition percentage $=($ activity of SjTGR - activity of SjTGR with isolated phage)/activity of SjTGR. The experiment was repeated three times.

The activities of TrxR, GR, and Grx of SjTGR were assayed with different concentrations $(0.5 \mu \mathrm{M}, 1 \mu \mathrm{M}$, and $2 \mu \mathrm{M})$ of the synthesized peptide JIPDys1 to determine the $50 \%$ inhibitory concentration $\left(\mathrm{IC}_{50}\right)$, and the same amount of PBS was added to the system as control. The experiment was repeated three times. The $\mathrm{IC}_{50}$ values were calculated by curve fitting using the SPSS 13.0 software.

2.5. Homologous Modeling of SjTGR. The dimer structure of SjTGR was constructed based on the known structure of SmTGR (PDB ID: 2x99 and 2x8c) [26] using software MODELLER 9v8 program [27-29]. Structural refinements were accomplished by energy minimization where the initial 3000 steps of steep descent were followed by 2000 steps of conjugate gradient. Subsequently, 200 ps molecular dynamics 
TABLE 1: Enrichment of positive phage clones by panning with SjTGR from Ph.D.-12 phage library.

\begin{tabular}{lcccc}
\hline Rounds & SjTGR $(\mu \mathrm{g} /$ plate $)$ & Phage input $(\mathrm{pfu})$ & Phage Recovery $(\mathrm{pfu})$ & Recovery rate \\
\hline 1 & 100 & $1.0 \times 10^{11}$ & $1.3 \times 10^{4}$ & $1.3 \times 10^{-9}$ \\
2 & 100 & $1.1 \times 10^{11}$ & $2.4 \times 10^{5}$ & $2.2 \times 10^{-8}$ \\
3 & 100 & $2.0 \times 10^{11}$ & $1.5 \times 10^{7}$ & $1.7 \times 10^{-7}$ \\
\hline
\end{tabular}

TABle 2: Consensus sequences of peptides of recombinant phage clones randomly selected through biopanning from the Ph.D.-12 phage display library.

\begin{tabular}{lcc}
\hline Phage display peptide & Sequence of peptide & Proportion \\
\hline JIPDys1 & WPHNWWPHFKVK & $26 / 58$ \\
JIPDys2 & LHAETRSAMHRT & $2 / 58$ \\
JIPDys3 & YTMPSLTLYAMG & $3 / 58$ \\
JIPDys4 & KHMHWHPPALNT & $4 / 58$ \\
\hline
\end{tabular}

was performed to equilibrate the structure at $300 \mathrm{~K}$ using the software Discovery studio 2.5 (Accelrys, San Diego, USA). The final structure was the three-dimensional structural model of dimer SjTGR.

2.6. Computer Simulation of the Conformations of Peptide JIPDys1. The initial three-dimensional structure of peptide JIPDys1 was built by Discovery studio 2.5. The MD calculation was carried out using the CHARMM force field. The SHAKE algorithm was applied to fix all covalent bonds containing a hydrogen atom allowing a 2 fs time step to be used in the integration of Newton's equations. The nonbonded interaction energies and forces were smoothly shifted to zero at $1.2 \mathrm{~nm}$. Before MD simulations were carried out, the peptide of the solvated system was optimized by 2000 steps of steepest descent energy minimization followed by 1000 steps of conjugate gradient energy minimization. After the system was heated up to $300 \mathrm{~K}$, the $10 \mathrm{~ns} \mathrm{MD}$ simulation at $300 \mathrm{~K}$ and $1 \mathrm{~atm}$ was carried out. The MD simulation was performed under periodic boundary conditions, and coordinates were saved every $10 \mathrm{ps}$. The structures of the peptide JIPDys1 during MD simulation were clustered into several different conformations according to RMSD (root mean square deviation).

2.7. Peptide JIPDys1 Docking with SjTGR. Different conformations of peptide JIPDys1 from the MD simulation were docked into SjTGR. The docking of the peptide to the SjTGR homology structure was performed using ZDOCK of Discovery studio 2.5, a rigid-body protein-protein docking software [30]. ZDOCK used a fast Fourier transformation to search all possible binding modes for the proteins, performing evaluation based on shape complementarity, desolvation energy, and electrostatics. The top predictions from ZDOCK were then recomputed by RDOCK to improve the energies and eliminate clashes.

2.8. Statistical Analysis. All data are given as the mean \pm standard deviation (SD). The activities of $\operatorname{TrxR}, \mathrm{GR}$, and

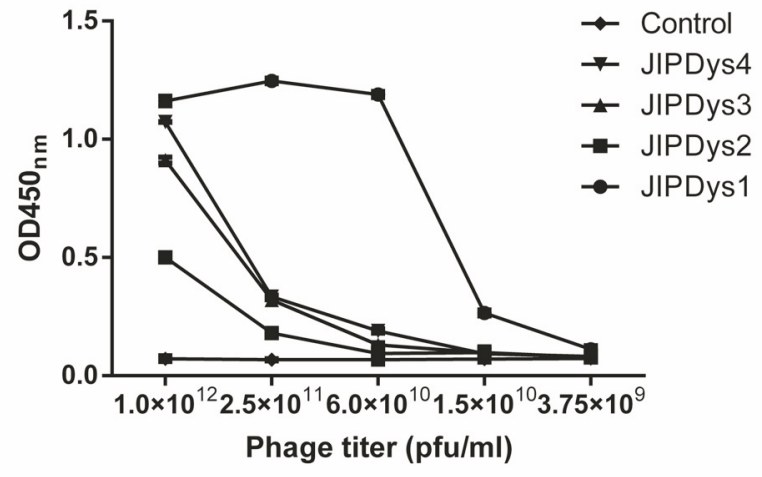

FIGURE 1: Binding of four selected phages to SjTGR determined by ELISA. The OD450nm values represent the binding ability of JIPDys1, JIPDys2, JIPDys3, and JIPDys4 and the negative phage control (phage library) at five different titers $\left(1.0 \times 10^{12}, 2.5 \times 10^{11}\right.$, $6.0 \times 10^{10}, 1.5 \times 10^{10}$, and $\left.3.75 \times 10^{9} \mathrm{pfu} / \mathrm{ml}\right)$. Binding decreased with decreasing titers of peptides. Results shown are the mean of triplicates \pm SD.

Grx of SjTGR (absorbance/S ( $\triangle \mathrm{A} / \mathrm{S})$ ) with JIPDys1, JIPDys2, JIPDys3, and JIPDys4 were statistically compared with the negative phage control (phage library) using two-tailed Student's t-tests. The activities of TrxR, GR, and Grx of SjTGR (absorbance/S $(\Delta \mathrm{A} / \mathrm{S})$ ) with the synthesized peptide were statistically compared with PBS control using two-tailed Student's t-tests, too. SPSS 13.0 was used for the statistical analyses. Differences between mean values were considered to be significant at the level of $5 \%$.

\section{Results}

3.1. Recombinant Phages and Binding to SjTGR by ELISA. Three rounds of biopanning enriched phages that bound well to $\mathrm{SjTGR}$ as indicated by the increased recovery (Table 1). Four consensus display peptides, named JIPDys1, JIPDys2, JIPDys3, and JIPDys4, were found in 58 clones of phages randomly selected for sequencing. Their aa sequences are shown in Table 2. Almost half (26 clones out of 58) carried gene sequences identical to JIPDys1.

The binding activity of the four peptides was detected by ELISA. The result (Figure 1) showed that at a concentration of $1 \times 10^{12} \mathrm{pfu} / \mathrm{ml}$ of all phages, JIPDys1, JIPDys2, JIPDys3, and JIPDys4, bound to SjTGR, although the binding capacity decreased with decreasing concentrations. Phage JIPDys1 displayed the strongest binding capacity among those phages. 


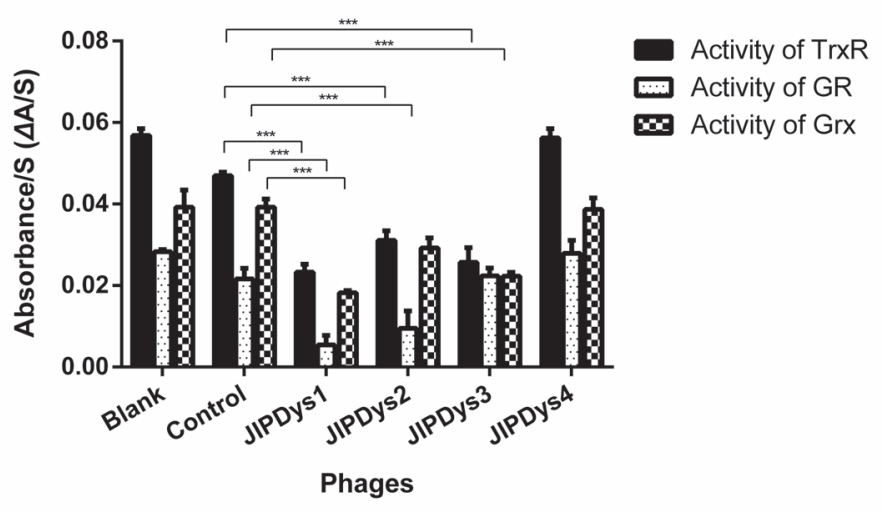

FIGURE 2: Inhibitory effect of four phages on the activity of TrxR, GR, and Grx of SjTGR. SjTGR was inhibited by $10^{11}$ pfu of JIPDys1, JIPDys2, and JIPDys3, while JIPDys4 was similar to the negative phage control (phage library). Blank means the activity of TrxR, GR, and Grx without phage. Results shown are the mean of triplicates $\pm \mathrm{SD}$. ${ }^{* * *}$ The difference was statistically significant $(P<0.01)$.

3.2. Inhibition of SjTGR by Phage Clones. An inhibition test using all four selected phage clones showed that at $10^{11} \mathrm{pfu}$ JIPDys1, JIPDys2, and JIPDys3 inhibited the activities of TrxR, GR, and Grx of SjTGR (Figure 2). The most powerful inhibition of enzyme activity was noted with JIPDysl; the percentages of inhibition of TrxR, GR, and Grx of SjTGR $(48 \mathrm{nM})$ were $59.04 \%, 80.80 \%$, and $53.56 \%$, respectively. Percentages of inhibition for JIPDys2 were $45.24 \%, 69.43 \%$, and $25.64 \%$; for JIPDys3 they were $54.72 \%, 20.88 \%$, and $43.02 \%$; and for JIPDys4 they were $0.73 \%, 1.50 \%$, and $1.23 \%$, respectively. Comparing the activity of TrxR, GR, and Grx of SjTGR with negative phage control (phage library) and JIPDysl, differences were statistically significant $(P<0.01)$. There were statistical differences in the activity of TrxR and GR of SjTGR between JIPDys2 and negative phage control $(P<0.01)$. And there were statistical differences in the activity of TrxR and Grx of SjTGR between JIPDys3 and negative phage control $(P<0.01)$.

3.3. Inhibition of SjTGR by Synthetic Peptide JIPDys1. As a result of its outstanding performance in initial experiments, the peptide JIPDys1 was synthesized artificially and used as an inhibitor in subsequent experiments. The results showed that $1 \mu \mathrm{M}$ of the synthesized peptide inhibited the activity of TrxR, GR, and Grx of SjTGR (48 nM) by $22.5 \%$, 88.5\%, and $49.1 \%$, and there were statistical differences in the activity of TrxR, GR, and Grx of SjTGR when compared without the synthesized peptide $(P<0.01) .2 \mu \mathrm{M}$ of the synthesized peptide inhibited the activity of TrxR, GR, and Grx of SjTGR ( $48 \mathrm{nM}$ ) by $32.5 \%, 100 \%$, and $100 \%$, respectively (Figure 3), and there were statistical differences in the activity of TrxR, GR, and Grx of SjTGR when compared without the synthesized peptide $(P<0.01)$. The $\mathrm{IC}_{50}$ values of the synthetic peptide JIPDys1 for TrxR, GR, and Grx were $3.67 \mu \mathrm{M}, 0.11$ $\mu \mathrm{M}$, and $0.97 \mu \mathrm{M}$, respectively.

3.4. Homologous Structure of SjTGR. The aa homology of SjTGR and SmTGR was 82\% [19]. SmTGR has TrxR and GR function, and its native structure is a homologous dimer. It

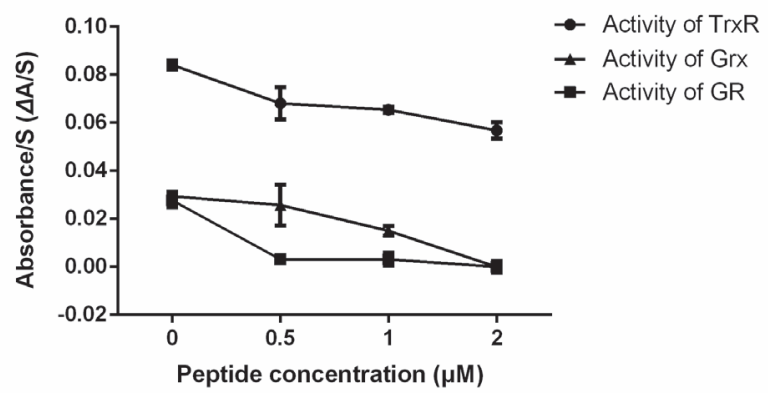

FIGURE 3: Effect of synthetic peptide on the activity of TrxR, GR, and Grx of SjTGR. SjTGR was inhibited in a concentration dependent manner by the synthesized peptide $(0.5 \mu \mathrm{M}, 1 \mu \mathrm{M}$, and $2 \mu \mathrm{M})$. Results shown are the mean of triplicates \pm SD.

was used as the template and in accordance with the structure of human oxidized glutathione (GSSG) (PDB ID 2GRT) we set up the dimer model for the SjTGR complex with FAD, NADPH, GSSG, and GSH (Figure 4(a)). The residues of SjTGR interacting with GSSG (the active sites of GR) and GSH (the active sites of Grx) are shown in Figure 4(b). The active site of the GR unit is located at the interface of the dimer, and the residues interacting with GSSG are Serl17, Leu120-Leu 124, Ile160, Leu163, Leu208, Tyr212, and Ile446 of one subunit and Pro507, Leu 508, His 571-Thr 580, and Val 593-Gly 595 of the other subunit. The Grx active domain is $\mathrm{N}$-terminal and its substrate, GSH, is surrounded by Lys25Phe30, Gln60, Thr71-Gln74, and Asp84-Lys86.

3.5. Computer Simulation of Binding between Peptide JIPDys1 and SjTGR. To obtain information on the mechanism of inhibition, interactions of peptide-protein were predicted using the software ZDOCK [29]. Typical conformations of JIPDysl predicted by MD simulation were selected to simulate docking with SjTGR.

Possible structures of JIPDys1 are shown in Figure 5(a). To find suitable binding sites, JIPDys1 was docked into the 


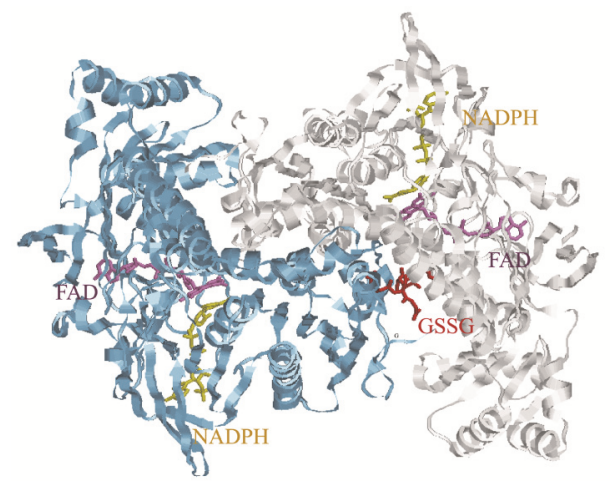

(a)
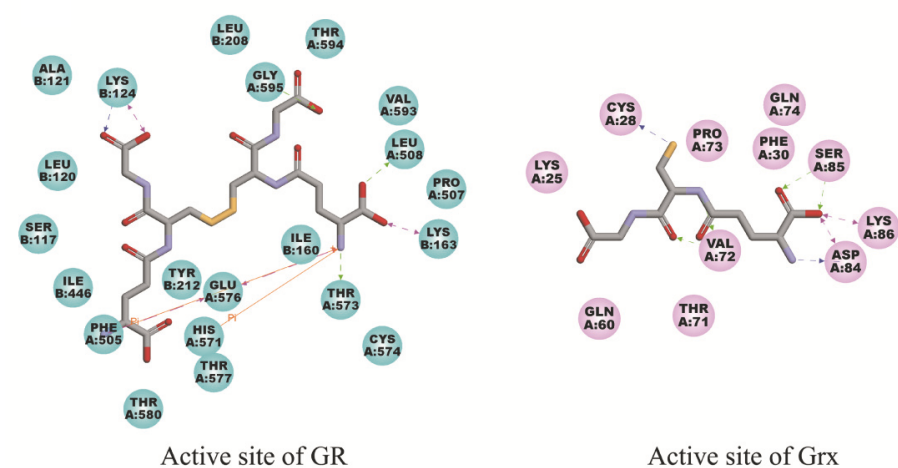

(b)

FIGURE 4: (a) The dimer structure model of SjTGR, including ligands of FAD, NADPH, and GSSG molecules. (b) aa of the GR domain interacting with GSSG (left) and of the Grx domain with GSH (right).

whole SjTGR. The structures with the highest values (top $10 \%)$ were selected for analysis. Figure 5(b) shows that the SjTGR residues that interacted with JIPDysl were 1-10, 2630 , 45-53, 60-63, 70-83, 124-129, 155, 160, 203-218, 262-255, 293-296, 322-324, 391-394, 420-423, 437-440, 450-463, 501518, 565-566, and 571-595, including the Grx domain, the NADPH binding site, and the GSSG active site. The region with the highest docking frequencies was identified at the GSSG binding site.

To explore the inhibition of GR activity by JIPDys1 in more detail, the peptide was docked directly into the dimer interface of SjTGR. The frequency of interaction between the residues of the GR domain in SjTGR and JIPDys1 is visualized in Figure 5(c). The values represent the probability of SjTGR residues binding to the peptide. This analysis indicated that the frequency of JIPDys1 interaction with Lys124, Leu208, Tyr212, Ser215, Ile446, and Arg 450 of one subunit and Ser503Leu508, His571, Glu576, Thr577, Thr580, His582, and Val593 of the other subunit of SjTGR was high, suggesting that these residues are most important for the stability of the interaction. In addition, JIPDys1 interacted with Cys154 and Cys159 of SjTGR, although with lower frequency.

Our early work demonstrated that JIPDys1 may inhibit the activity of Grx, and this was supported by simulations which indicated JIPDys1 docking at the GSH binding site of Grx (Figure 5(d)). The result showed high interaction frequencies of JIPDys1 with Phe30, Gln60, Lys68-Val72, and Asp84-Val88 of SjTGR, which are the most important residues for GSH interaction with the Grx domain (Figure 5(e)).

\section{Discussion}

Peptide drugs have aroused general interest for the development of novel drugs due to their ease of usage, fast absorption, and lack of side effects. To date, research exploring peptide drugs has mainly concentrated on tumor treatment, cardiovascular, viral, and microbial diseases, and corneal limbal epithelial stem cell deficiency [31-33]. In addition, development of powerful screening technologies such as phage display library has provided means that have been used in ligand mapping to define peptides that bind to a given antibody or receptor molecule. For example, Sperinde [34] has identified a peptide which could inhibit the activity of DNase II using a circular 12-phage display peptide library. Similarly, Dennis [35] obtained a peptide inhibiting the activity of serine protease by screening a phage display library.

We have previously shown [21] that SjTGR plays an essential role in maintaining the redox balance in $S$. japonicum, which suggested TGR as a potential target for the development of new drugs against schistosomiasis. TGR were detected in the tegument of worms [36]. Peptide drugs 


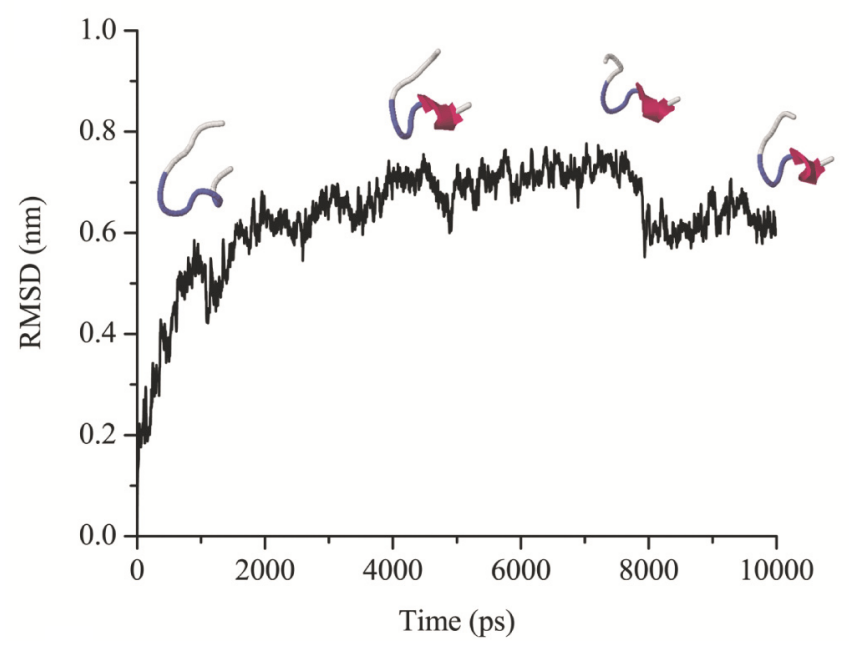

(a)
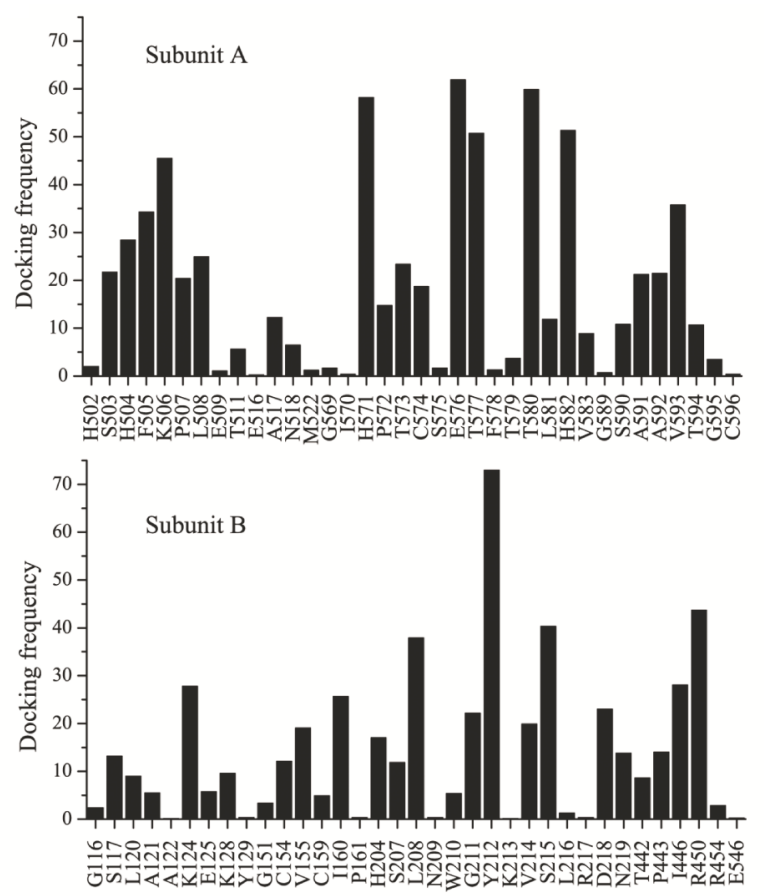

Amino acids of GR active site interacting with the peptide

(c)

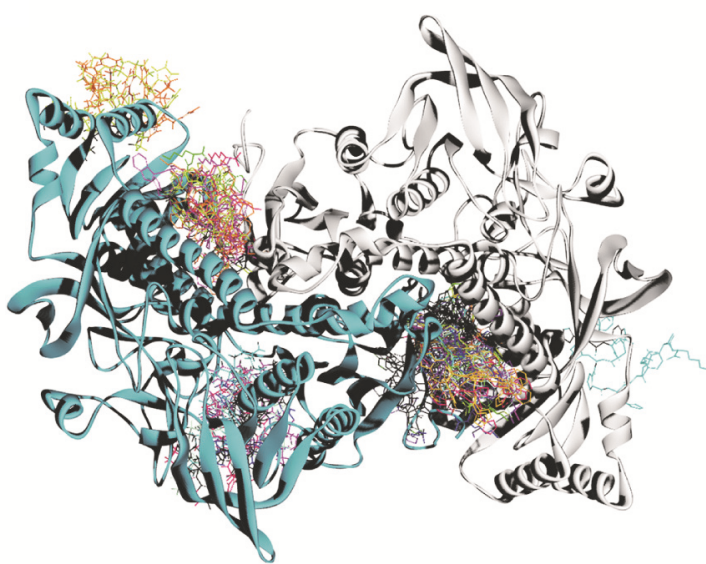

(b)

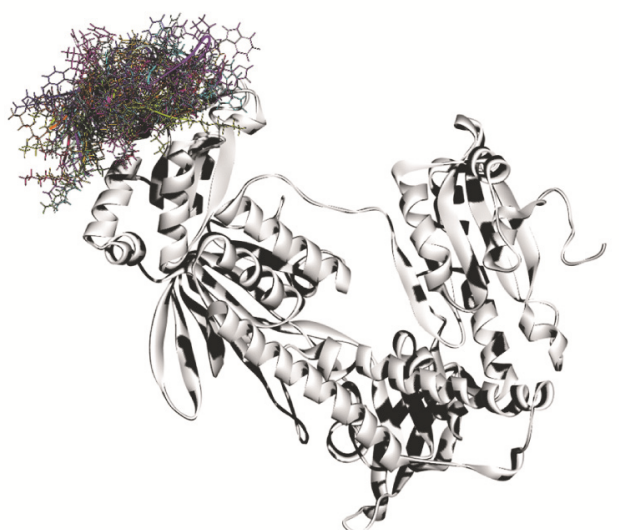

(d)

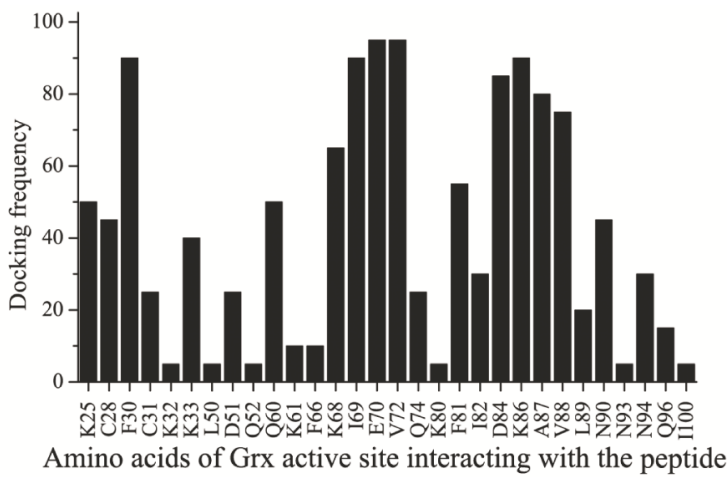

(e)

FIGURE 5: (a) Selected conformations of JIPDysl during molecular dynamics. (b) Overlap of the peptide JIPDys1 docking with TGR. The regions of SjTGR that interacted with JIPDysl included the Grx domain, the NADPH binding unit, and the GSSG binding site. The region of high docking frequency centralized mainly at GSSG binding site. (c) Frequency distribution of JIPDys1 docking to the GR active site of SjTGR. (d) Overlap of docked JIPDys1 at the GSH binding site of the Grx domain. (e) Docking frequency of residues at the Grx active site.

against TGR could reach the worm in the blood vessel by intravenous injection. The worm may be dead due to the oxidative damage from the host. Our current work confirms this approach as viable through the identification of a peptide with strong inhibitory activity of SjTGR. Out of the four consensus sequences, JIPDys1, JIPDys2, JIPDys3, and JIPDys4, that were identified by screening a Ph.D. ${ }^{\mathrm{TM}}-12$ phage display peptide library, three (JIPDys1, JIPDys2, and JIPDys3) showed inhibitory activity to SjTGR. Among these, JIPDysl inhibited the activity by more than $50 \%$.

In blast searches using NCBI, the aa sequence of JIPDys1 was $78 \%$ identical to the $\mathrm{NAD}(\mathrm{P})$ binding region, and 
JIPDys 2 and JIPDys3 were $89 \%$ and $64 \%$ similar to hypothetical proteins (data not shown). These results suggested that the binding sites and potentially the mechanism of inhibition of JIPDys 2 and JIPDys3 were different from those of JIPDys1.

The synthetic peptide JIPDysl displayed the strongest inhibitory effect on the activity of SjTGR, in a dose dependent fashion. Its inhibitory effect was more pronounced on GR and Grx than TrxR. This is consistent with the results of a molecular docking computer simulation which suggested binding of the peptide to the substrate binding sites of GR and Grx. The region with the highest docking frequencies was identified at the GSSG binding site. Residues in SjTGR that were identified to have high interaction frequency with JIPDys1 are the important aa binding to GSSG. Therefore, JIPDys1 could interfere with GSSG entering into its binding sites, competitively inhibiting the GR activity of SjTGR. The interaction between JIPDys1 and residues of the domain related to TrxR function was not powerful. Although JIPDys1 interacted with Cys154 and Cys159 of SjTGR, the frequency was much lower. Cys154 and Cys159 are related to the electron delivery system of TrxR, and this may be the reason that the inhibitory effect of JIPDys1 on TrxR was not obvious. To enhance the inhibitory effect of the peptide JIPDysl on the TrxR activity of SjTGR, it would be necessary to remodel the structure of the peptide JIPDys1. This may allow designing a peptide that would inhibit the activity of TrxR, GR, and Grx of SjTGR.

Based on our results one could think of ways to improve the activity of a potential peptide drug by optimizing the aa content of JIPDysl by adding residues that would bind to the active centers of SjTGR strongly, by reducing hydrophobic aa to increase solubility in water, or by designing novel peptide antagonists of SjTGR by computer simulation and molecular docking taking advantage of the features of JIPDys.

\section{Conclusion}

Taking all together, immune binding, enzyme activity, and computer simulation provide evidence that a novel peptide, JIPDys1 (aa, WPHNWWPHFKVK), could bind with SjTGR and reduce enzyme activity of SjTGR, which is a potential candidate to develop novel drugs against S. japonicum. The peptide drugs are easily decomposed by protease in vivo, so the structure of peptides needs to be modified by using drug delivery system, which should be studied further. The research makes a foundation for studying peptide drugs against schistosomiasis or provides a new direction for development of the novel drugs against Schistosoma infection.

\section{Data Availability}

The datasets used and analyzed during the current study are available from the corresponding author on reasonable request.

\section{Disclosure}

The manuscript was presented as an abstract in the 10th National Symposium on Parasitology.

\section{Conflicts of Interest}

The authors declare no conflicts of interest.

\section{Authors' Contributions}

Li-Jun Song designed and performed the study, managed, analyzed, and interpreted the data, and prepared the manuscript; Jia-Huang Li designed the study and facilitated and assisted the study implementation; Xu-Ren Yin, Wei Zhang, and Yi Jin assisted in the design and study implementation and revised the manuscript; Hong Gao and Jie Wang assisted in the design of the study and data analysis; Chuan$\mathrm{Xin} \mathrm{Yu}$ and $\mathrm{Zi}$-Chun Hua designed the study, supervised the study implementation, and revised the manuscript. All authors read and approved the final manuscript. Li-Jun Song and Jia-Huang Li contributed equally to this work.

\section{Acknowledgments}

This work was supported by grants from the National Natural Science Foundation of China (81573338, 81630092, 81570790, and 81773099), the Natural Science Foundation of Jiangsu Province (BK2012544, BZ2017018, and BK20151457), the Scientific Research Projects from Jiangsu Provincial Commission of Health and Family Planning (H201635), the Scientific Research Projects from Wuxi City Commission of Health and Family Planning (Q201656), the Jiangsu Provincial Project of Invigorating Health Care through Science, Technology and Education, Jiangsu Science and Technology Department (no. BM2015024), and Shenzhen Science and Technology Innovation Committee (JCYJ20160331152141936, KQTD20140630165057031).

\section{References}

[1] P. Steinmann, J. Keiser, R. Bos, M. Tanner, and J. Utzinger, "Schistosomiasis and water resources development: systematic review, meta-analysis, and estimates of people at risk," The Lancet Infectious Diseases, vol. 6, no. 7, pp. 411-425, 2006.

[2] M. J. Van Der Werf, S. J. De Vlas, S. Brooker et al., "Quantification of clinical morbidity associated with schistosome infection in sub-Saharan Africa," Acta Tropica, vol. 86, no. 2-3, pp. 125139, 2003.

[3] C. H. King, K. Dickman, and D. J. Tisch, "Reassessment of the cost of chronic helmintic infection: a meta-analysis of disabilityrelated outcomes in endemic schistosomiasis," The Lancet, vol. 365, no. 9470, pp. 1561-1569, 2005.

[4] G. Andrade, D. J. Bertsch, A. Gazzinelli, and C. H. King, "Decline in infection-related morbidities following drugmediated reductions in the intensity of Schistosoma infection: a systematic review and meta-analysis," PLoS Neglected Tropical Diseases, vol. 11, no. 2, Article ID e0005372, 2017.

[5] Y. Osada and T. Kanazawa, "Schistosome: its benefit and harm in patients suffering from concomitant diseases," Journal of Biomedicine and Biotechnology, vol. 2011, Article ID 264173, 10 pages, 2011.

[6] J. R. Coura and M. J. Conceição, "Specific schistosomiasis treatment as a strategy for disease control," Memórias do Instituto Oswaldo Cruz, vol. 105, no. 4, pp. 598-603, 2010. 
[7] WHO Expert Committee, "Prevention and control of schistosomiasis and soil transmitted helminthiasis," World Health Organization Technical Report Series 912, 2002.

[8] P. G. Fallon, R. F. Sturrock, A. Capron, M. Niang, and M. J. Doenhoff, "Short report: diminished susceptibility to praziquantel in a Senegal isolate of Schistosoma mansoni," The American Journal of Tropical Medicine and Hygiene, vol. 53, no. 1, pp. 61-62, 1995.

[9] M. Ismail, A. Metwally, A. Farghaly, J. Bruce, L.-F. Tao, and J. L. Bennett, "Characterization of isolates of Schistosoma mansoni from Egyptian villagers that tolerate high doses of praziquantel," The American Journal of Tropical Medicine and Hygiene, vol. 55, no. 2, pp. 214-218, 1996.

[10] L. A. Tchuem Tchuenté, V. R. Southgate, A. Mbaye, D. Engels, and B. Gryseels, "The efficacy of praziquantel against Schistosoma mansoni infection in Ndombo, northern Senegal," Transactions of the Royal Society of Tropical Medicine and Hygiene, vol. 95, no. 1, pp. 65-66, 2001.

[11] A. M. B. Mendonça, A. P. S. Feitosa, D. L. Veras et al., "The susceptibility of recent isolates of Schistosoma mansoni to praziquantel," Revista do Instituto de Medicina Tropical de São Paulo, vol. 58, p. 7, 2016.

[12] S. D. Melman, M. L. Steinauer, C. Cunningham et al., "Reduced susceptibility to praziquantel among naturally occurring Kenyan isolates of Schistosoma mansoni," PLoS Neglected Tropical Diseases, vol. 3, no. 8, article e504, 2009.

[13] D. Alonso, J. Muñoz, J. Gascón, M. E. Valls, and M. Corachan, "Short report: failure of standard treatment with praziquantel in two returned travelers with Schistosoma haematobium infection," The American Journal of Tropical Medicine and Hygiene, vol. 74, no. 2, pp. 342-344, 2006.

[14] I. M. Da Silva, R. Thiengo, M. J. Conceição et al., "Therapeutic failure of praziquantel in the treatment of Schistosoma haematobium infection in Brazilians returning from Africa," Memórias do Instituto Oswaldo Cruz, vol. 100, no. 4, pp. 445-449, 2005.

[15] J. Utzinger, X. Shuhua, E. K. N'Goran, R. Bergquist, and M. Tanner, "The potential of artemether for the control of schistosomiasis," International Journal for Parasitology, vol. 31, no. 14, pp. 1549-1562, 2001.

[16] A. Holmgren, "Thioredoxin and glutaredoxin systems," The Journal of Biological Chemistry, vol. 264, no. 24, pp. 1396313966, 1989.

[17] D. Cioli, C. Valle, F. Angelucci, and A. E. Miele, "Will new antischistosomal drugs finally emerge?" Trends in Parasitology, vol. 24, no. 9, pp. 379-382, 2008.

[18] K. Becker, S. Gromer, R. Heiner Schirmer, and S. Müller, "Thioredoxin reductase as a pathophysiological factor and drug target," European Journal of Biochemistry, vol. 267, no. 20, pp. 6118-6125, 2000.

[19] A. N. Kuntz, E. Davioud-Charvet, A. A. Sayed et al., "Thioredoxin glutathione reductase from Schistosoma mansoni: an essential parasite enzyme and a key drug target," PLoS Medicine, vol. 4, article e206, 2007.

[20] A. A. Sayed, A. Simeonov, C. J. Thomas, J. Inglese, C. P. Austin, and D. L. Williams, "Identification of oxadiazoles as new drug leads for the control of schistosomiasis," Nature Medicine, vol. 14, no. 4, pp. 407-412, 2008.

[21] L. Song, J. Li, S. Xie et al., "Thioredoxin glutathione reductase as a novel drug target: evidence from Schistosoma japonicum," PLoS ONE, vol. 7, no. 2, Article ID e31456, 2012.

[22] J. J. Martínez-González, A. Guevara-Flores, G. Álvarez, J. L. Rendón-Gómez, and I. P. Del Arenal, "In vitro killing action of auranofin on Taenia crassiceps metacestode (cysticerci) and inactivation of thioredoxin-glutathione reductase (TGR)," Parasitology Research, vol. 107, no. 1, pp. 227-231, 2010.

[23] A. Agorio, C. Chalar, S. Cardozo, and G. Salinas, "Alternative mRNAs arising from trans-splicing code for mitochondrial and cytosolic variants of Echinococcus granulosus thioredoxin glutathione reductase," The Journal of Biological Chemistry, vol. 278, no. 15, pp. 12920-12928, 2003.

[24] A. Guevara-Flores, J. P. Pardo, and J. L. Rendón, "Hysteresis in thioredoxin-glutathione reductase (TGR) from the adult stage of the liver fluke Fasciola hepatica," Parasitology International, vol. 60, no. 2, pp. 156-160, 2011.

[25] G. Kubas, W. Rees, J. Caguiat, D. Asch, D. Fagan, and P. Cortes, "Identification of peptide sequences that selectively bind to pentaerythritol trinitrate hemisuccinate-a surrogate of PETN, via phage display technology," Biopolymers, vol. 108, no. 2, Article ID e22997, 2017.

[26] F. Angelucci, D. Dimastrogiovanni, G. Boumis et al., "Mapping the catalytic cycle of Schistosoma mansoni thioredoxin glutathione reductase by X-ray crystallography," The Journal of Biological Chemistry, vol. 285, no. 42, pp. 32557-32567, 2010.

[27] M. A. Marti-Renom, A. C. Stuart, A. Fiser, R. Sanchez, F. Melo, and A. Sali, "Comparative protein structure modeling of genes and genomes," Annual Review of Biophysics, vol. 29, pp. 291-325, 2000.

[28] A. Sali and T. L. Blundell, "Comparative protein modelling by satisfaction of spatial restraints," Journal of Molecular Biology, vol. 234, no. 3, pp. 779-815, 1993.

[29] A. Fiser, R. Kinh Gian Do, and A. Sali, "Modeling of loops in protein structures," Protein Science, vol. 9, no. 9, pp. 1753-1773, 2000.

[30] J. Mintseris, B. Pierce, K. Wiehe, R. Anderson, R. Chen, and Z. Weng, "Integrating statistical pair potentials into protein complex prediction," Proteins: Structure, Function, and Genetics, vol. 69, no. 3, pp. 511-520, 2007.

[31] S. Rita, S. Anna, and S. Susanna, "Effects of synthetic peptides on the inflammatory response and their therapeutic potential," Mini-Reviews in Medicinal Chemistry, vol. 13, no. 4, pp. 553-564, 2013.

[32] E. Fok, S. R. Sandeman, A. L. Guildford, and Y. H. Martin, "The use of an IL-1 receptor antagonist peptide to control inflammation in the treatment of corneal limbal epithelial stem cell deficiency," BioMed Research International, vol. 2015, Article ID 516318, 14 pages, 2015.

[33] C. Polanco, J. L. Samaniego, T. Buhse et al., "Characterization of selective antibacterial peptides by polarity index," International Journal of Peptides, vol. 2012, Article ID 585027, 11 pages, 2012.

[34] J. J. Sperinde, S.-J. Choi, and F. C. Szoka Jr., "Phage display selection of a peptide DNase II inhibitor that enhances gene delivery," The Journal of Gene Medicine, vol. 3, no. 2, pp. 101-108, 2001.

[35] M. S. Dennis, C. Elgenbrot, N. J. Skelton et al., "Peptide exosite inhibitors of factor VIIa as anticoagulants," Nature, vol. 404, no. 6777, pp. 465-470, 2000.

[36] Y. Han, M. Zhang, Y. Hong et al., "Characterization of thioredoxin glutathione reductase in Schiotosoma japonicum," Parasitology International, vol. 61, no. 3, pp. 475-480, 2012. 


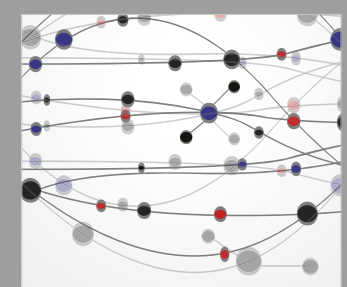

The Scientific World Journal
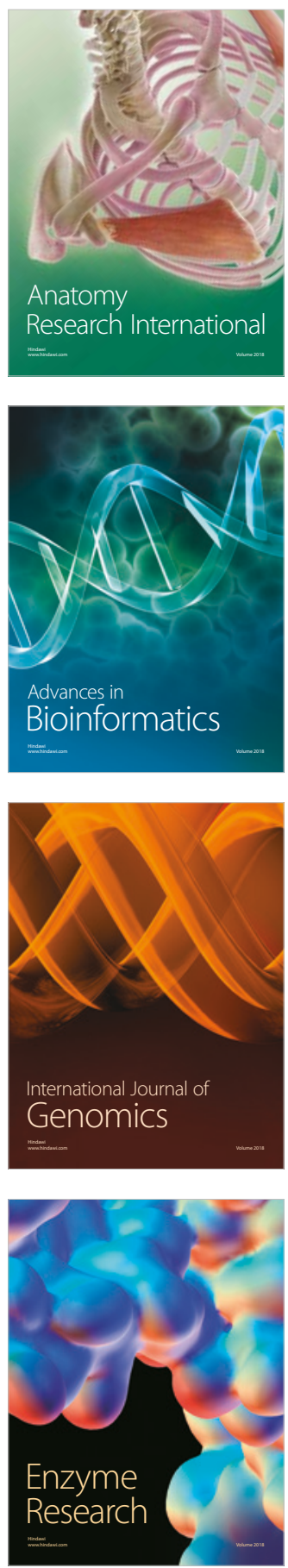
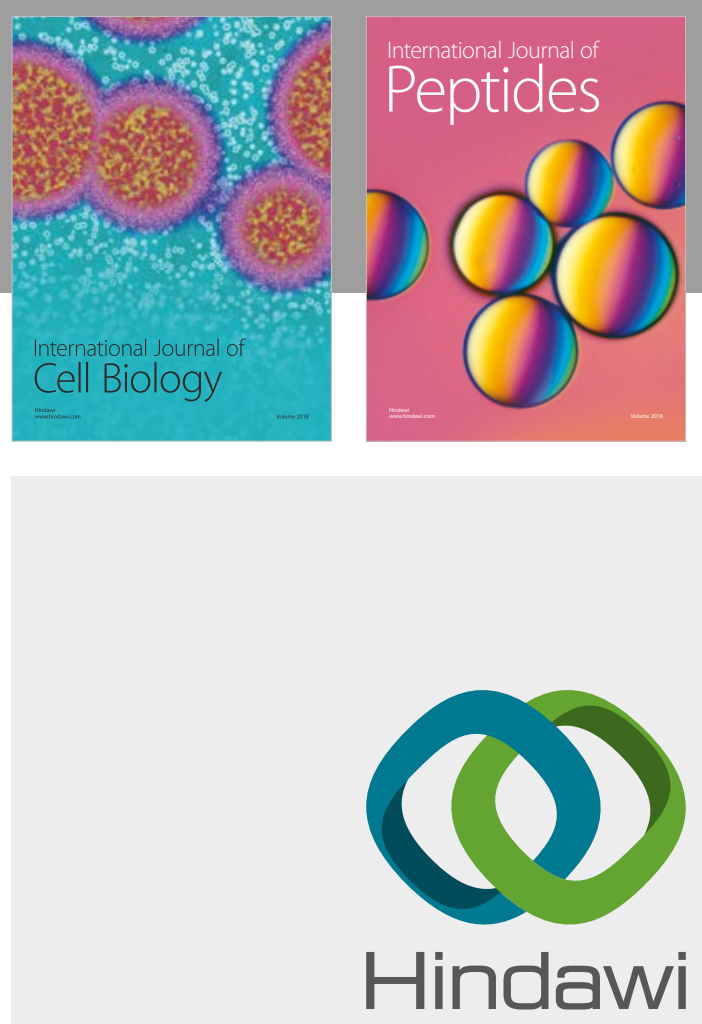

Submit your manuscripts at

www.hindawi.com
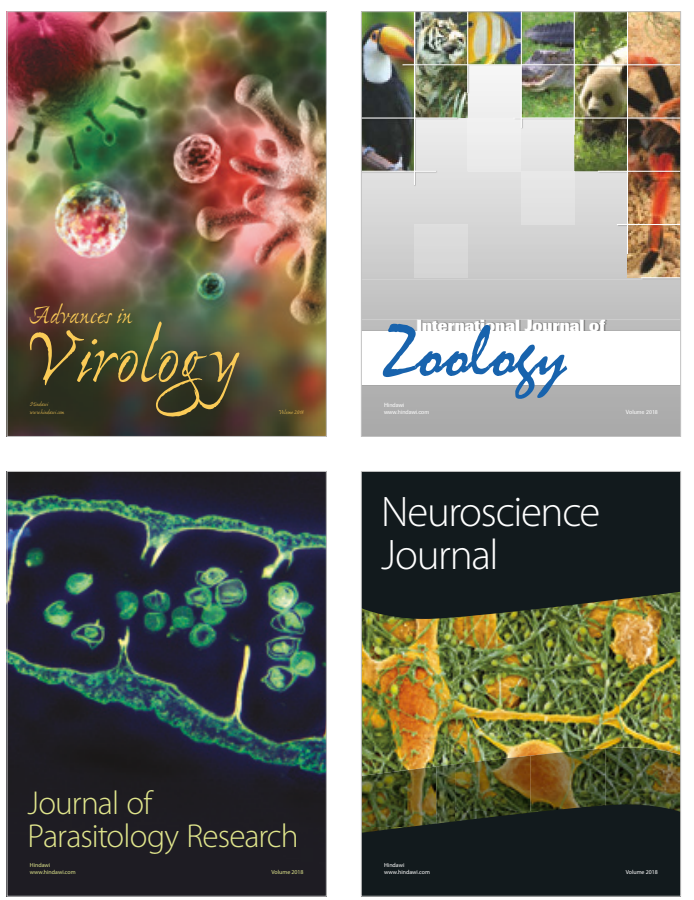
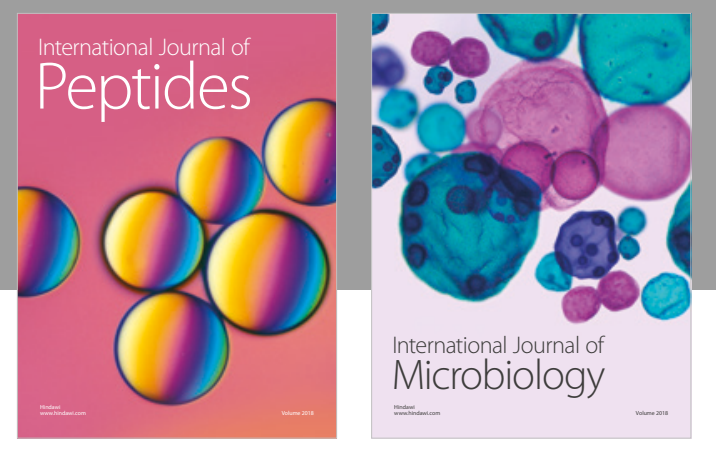

nternational Journal of Microbiology
Journal of
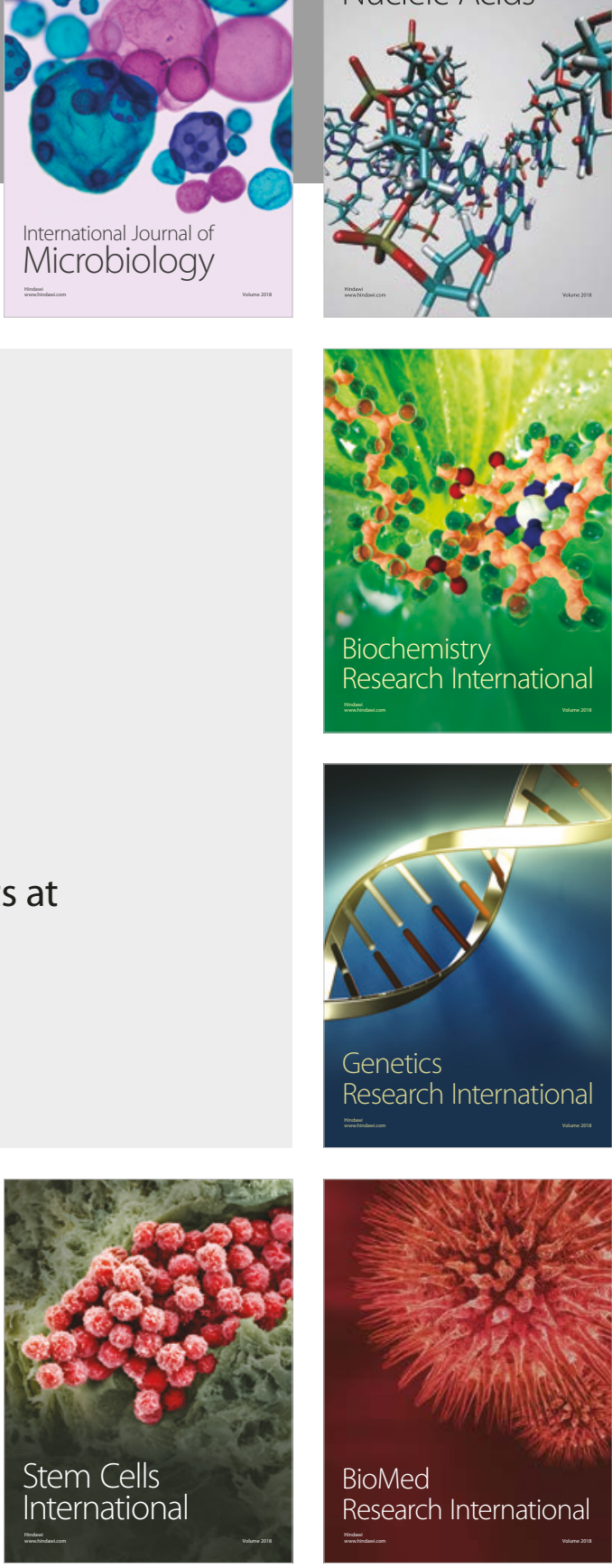
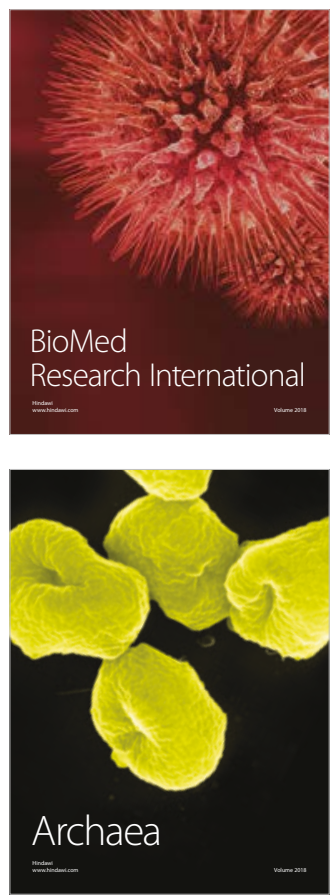